\title{
Pharmacogenomics in diabetes mellitus insights into drug action and drug discovery
}

Zhou, Kaixin; Pedersen, Helle Krogh; Dawed, Adem Y.; Pearson, Ewan R.

\section{Published in:}

Nature Reviews. Endocrinology

Link to article, DOI:

10.1038/nrendo.2016.51

Publication date:

2016

Document Version

Peer reviewed version

Link back to DTU Orbit

Citation (APA):

Zhou, K., Pedersen, H. K., Dawed, A. Y., \& Pearson, E. R. (2016). Pharmacogenomics in diabetes mellitus: insights into drug action and drug discovery. Nature Reviews. Endocrinology, 12(6), 337-346.

https://doi.org/10.1038/nrendo.2016.51

\section{General rights}

Copyright and moral rights for the publications made accessible in the public portal are retained by the authors and/or other copyright owners and it is a condition of accessing publications that users recognise and abide by the legal requirements associated with these rights.

- Users may download and print one copy of any publication from the public portal for the purpose of private study or research.

- You may not further distribute the material or use it for any profit-making activity or commercial gain

- You may freely distribute the URL identifying the publication in the public portal

If you believe that this document breaches copyright please contact us providing details, and we will remove access to the work immediately and investigate your claim. 


\section{OPEN ACCESS}

University of Dundee

\section{Pharmacogenomics in diabetes mellitus}

Zhou, Kaixin; Pedersen, Helle Krogh; Dawed, Adem Y; Pearson, Ewan R

Published in:

Nature Reviews Endocrinology

DOI:

10.1038/nrendo.2016.51

Publication date:

2016

Document Version

Peer reviewed version

Link to publication in Discovery Research Portal

Citation for published version (APA):

Zhou, K., Pedersen, H. K., Dawed, A. Y., \& Pearson, E. R. (2016). Pharmacogenomics in diabetes mellitus: insights into drug action and drug discovery. Nature Reviews Endocrinology, 12(6), 337-346. DOI:

$10.1038 /$ nrendo.2016.51

\section{General rights}

Copyright and moral rights for the publications made accessible in Discovery Research Portal are retained by the authors and/or other copyright owners and it is a condition of accessing publications that users recognise and abide by the legal requirements associated with these rights.

- Users may download and print one copy of any publication from Discovery Research Portal for the purpose of private study or research. - You may not further distribute the material or use it for any profit-making activity or commercial gain.

- You may freely distribute the URL identifying the publication in the public portal.

\section{Take down policy}

If you believe that this document breaches copyright please contact us providing details, and we will remove access to the work immediately and investigate your claim. 
Nature Reviews Endocrinology 12, 337-346 (2016) - http://www.nature.com/nrendo/journal/v12/ n6/full/nrendo.2016.51.html DOI:10.1038/nrendo.2016.51

\title{
Pharmacogenomics in diabetes mellitus - insights into drug action and drug discovery
}

\author{
Kaixin Zhou' ${ }^{1}$, Helle Krogh Pedersen ${ }^{2}$, Adem Y. Dawed ${ }^{1}$, Ewan R. Pearson ${ }^{1}$ \\ ${ }^{1}$ School of Medicine, University of Dundee, Dundee DD1 9SY, UK \\ ${ }^{2}$ Department of Systems Biology, Technical University of Denmark, 2800 Lyngby, Denmark \\ Correspondence to \\ E.R.P \\ e.z.pearson@dundee.ac.uk
}

Abstract | Genomic studies have greatly advanced our understanding of the multifactorial aetiology of type 2 diabetes mellitus (T2DM) as well as the multiple monogenic diabetes subtypes. In this Review, we discuss the existing pharmacogenetic evidence in both monogenic diabetes and T2DM, highlight the mechanistic insights from the study of side effects to antidiabetic drugs as well as their efficacy. The identification of extreme sulfonylurea sensitivity in patients with diabetes mellitus caused by heterozygous mutations in HNF1A represents a clear example of how pharmacogenetics can impact on patient care. However pharmacogenomic studies of response to antidiabetic drugs in T2DM has yet to translate into clinical practice, although some moderate genetic effects have now been described that merit follow up in genotype selected trials. We also discuss how future pharmacogenomic findings could provide insights into treatment response in diabetes that, complementary to other areas of human genetics, facilitates drug discovery and drug development for T2DM.

\section{Introduction}

In the past decade, genome-wide association studies (GWAS) and high-throughput sequencing, propelled by the fast development in affordable genomic technologies, have greatly advanced our understanding of the genetic aetiology of many common diseases ${ }^{1}$. Pharmacogenomic studies applying these genome-wide approaches to investigate drug response have also yielded important results ${ }^{2,3}$. In this Review, and in this context, we discuss the genomic evidence that has strengthened our understanding of the multifactorial aetiology of type 2 diabetes mellitus (T2DM) and discuss the emerging evidence that a complex genetic architecture might underline the variation in response to antidiabetic drugs. 
Genetic evidence in disease genomics is increasingly being used for target validation in drug discovery. We anticipate how robust pharmacogenomic evidence could provide more valuable information to predict both on-target and off-target effects in drug discovery and development.

\section{The multifactorial aetiology of T2DM}

T2DM is a complex metabolic disease characterized by hyperglycaemia resulting from functional impairment in insulin secretion, insulin action or both ${ }^{4}$. Both insulin resistance and secretory deficiency arise through the interplay of genetic and environmental risk factors ${ }^{5}$. GWAS, which have interrogated all the common genetic variants (minor allele frequency greater than 5\%), have identified $>120$ T2DM risk loci6,7. High-throughput sequencing studies, which could theoretically examine all the variants in the genome or at least the section that encodes proteins, have also enabled the discovery of rare variants (minor allele frequency $<5 \%$ ) at GWAS identified loci and novel loci for $\mathrm{T}_{2} \mathrm{DM}^{8,9}$. Together these common variants with small to moderate effects and rare variants with relatively large impacts could account for $\sim 15 \%$ of the total risk of developing T2DM and confirm its nature as a multisystem disorder ${ }^{6,10}$.

Glycaemic control is a key focus in the management of T2DM, and is associated with both microvascular and macrovascular benefits ${ }^{11-13}$. The treatment of T2DM has evolved with our understanding of the pathophysiology of this complex disease ${ }^{5}$. A wide range of drug treatment, characterized by different mechanism of action, is available to achieve glycaemic control in patients with T2DM (Figure 1) ${ }^{14}$. Apart from insulin replacement, traditional oral agents include the secretagogues that stimulate the pancreas to release insulin and the sensitizers that enhance the efficacy of insulin action ${ }^{14}$. New agents include the dipeptidylpeptidase-4 (DPP4) inhibitors, also known as the gliptins, that enhance the socalled 'incretin effect' and promote glucose-stimulated insulin secretion ${ }^{15}$; as well as the sodium-glucose cotransporter-2 (SGLT-2) inhibitors that reduce hyperglycaemia by increasing glucose elimination via the urine ${ }^{16}$. Although these drugs are all effective at lowering glucose in patients with T2DM, glycaemic control often fails even after a combination of the available treatment options due to the progressive nature of the disease.

Diabetes drug response can be considered at many levels, as outlined in Table 1, including the physiological response to the drug, or the long term effect of the drug in terms of microvascular or macrovascular risk reduction. In this Review, when considering drug response, we focus primarily on the glycaemic effect of drugs as this outcome has been the most studied. 


\section{Monogenic diabetes mellitus}

With the increasing awareness that T2DM is highly heterogeneous, and as we understand more about the aetiology of the disease, we can begin to subdivide 'T2DM' into distinct aetiological subtypes. This development can be seen with the increasing identification of monogenic forms of the disease, which until the past 10-15 years were misclassified as type 1 diabetes mellitus or T2DM. Understanding these aetiological subtypes has resulted in some of the earliest studies that provided the most clinically robust examples of pharmacogenetics to date. For example, patients with Maturity Onset Diabetes of the Young owing to mutations in HNF1A (which accounts for $\sim 3 \%$ of all diabetes mellitus cases diagnosed under the age of 30 years) are extremely sensitive to sulfonylurea treatment, and can successfully transition off insulin treatment ${ }^{17}$. Similarly patients with neonatal diabetes due to $K C N J 11$ or $A B C C 8$ mutations who have insulin dependent diabetes mellitus from soon after birth have been shown to respond to high dose sulfonylureas and to be able to transition off insulin onto oral sulfonylurea treatment ${ }^{18}$. These examples highlight how increasing awareness of aetiological subtypes of diabetes will enable a precise approach to treatment of diabetes mellitus and is an area of great interest. However, for the remainder of this review, we will focus on polygenic influences on drug response in T2DM.

\section{Pharmacogenomics and genetic architecture}

Pharmacogenetics aims to seek the genetic explanation of why individuals respond differently to drugs, both in terms of therapeutic efficacy as well as adverse drug reactions $(A D R)^{19}$. Prior to the emergence of genome wide genotyping arrays, pharmacogenetic studies focused on candidate genes with known links to drug distribution, metabolism or response pathways ${ }^{19}$. With the development of cost-effective genomic technologies, genome-wide genotyping and sequencing has transformed this traditional pharmacogenetic approach into a more global pharmacogenomic approach that can systematically interrogate millions of genetic polymorphisms across the genome ${ }^{20,21}$. Most published genome-wide studies of drug response are GWAS, and only a few such studies reported sequencingbased investigations. One example of a sequencing-based study is the use of publicly available whole-genome sequence data on 482 samples to profile 231 pharmacogenetic genes $^{22}$. In the same study, the authors also performed whole genome sequencing on 7 family members to try to explain the genetic basis of their variable response to anticoagulation treatment. The two terms pharmacogenetics and pharmacogenomics are often used interchangeably, but in this Review we use pharmacogenomics to refer to studies using genome-wide approaches. 
Biomarker discovery for precision medicine remains the long-term goal of pharmacogenomic studies. However, an often under-appreciated benefit of such studies is that they can advance our understanding of the biological mechanism of drug action in humans by identifying variants in genes not previously thought to be associated with drug response. These genes might never have been included in traditional candidate gene approaches ${ }^{3}$.

A fundamental issue underlying the validity and feasibility of pharmacogenomic studies is the genetic architecture of drug response ${ }^{23}$. In this context 'genetic architecture' refers to the number of response variants; the frequency spectrum of these response variants; the effect size spectrum of the variants; the physical distribution of the variants in the genome; and the amount of variation in drug response explained by these genetic variants (known as heritability). Whilst heritability determines the validity of pharmacogenomic studies, the other aspects of genetic architecture dictate the feasibility and design of pharmacogenomic studies.

Adopting traditional twin and family study designs to estimate the heritability of drug treatment outcomes has been largely impractical, because family members may not develop the same disease or be treated with same drug. With the availability of GWAS data, new 'chip-based' approaches have been developed to estimate heritability from population-based samples ${ }^{24}$. However, data from at least a few thousand individuals are required to achieve an accurate estimate of heritability by these methods ${ }^{25}$. Such methods, therefore, can be applied to estimate the heritability of treatment efficacy for commonly used drugs, but not the less frequent ADRs.

In a study of GWAS data from 2,085 patients with T2DM, heritability of glycaemic response to metformin was estimated to be up to $34 \%(p=0.02)^{23}$. Furthermore, this investigation also found that the heritability is likely to be the result of many common response variants with small to moderate effect sizes scattered across the genome ${ }^{23}$. These results suggest that the genetic architecture of metformin efficacy is similar to that of T2DM and other complex traits. This similarity between the genetic architectures of T2DM and the treatment efficacy of metformin is likely to be rooted in the multifactorial aetiology of the disease. Variants in different genes or pathways might affect metformin treatment efficacy in patients whose pathophysiology is heterogeneous (for example, those individuals who are predominantly insulin resistant or those whose insulin secretion is deficient). Similar to metformin, other antidiabetic agents are also used to treat a combination of patients with heterogeneous pathophysiology. Therefore we anticipate that the genetic architecture of treatment efficacy for other antidiabetic agents to be similar to that of metformin response. 
To appreciate the scale of the heritability estimate of $34 \%$ for glycaemic response to metformin, it is necessary to put it into the context of other complex traits. In a 2015 study again using a population-based method, the heritability estimate for BMI was $27 \%(\text { S.E. }=2.5 \%)^{26}$, which is considerably lower than the heritability estimates of $40 \% \sim 60 \%$ derived from traditional twin and family studies ${ }^{27}$. The discrepancy observed between the two methods could be explained by the facts that heritability is underestimated by the "chipbased' method due to imperfect tagging and it is often overestimated by the traditional twin studies due to common environment confounding ${ }^{28}$. Therefore the actual heritability of glycaemic response to metformin could be even higher than what has been estimated from GWAS data. In addition, chip-based heritability estimates also suffer underestimation due to the incomplete coverage of contribution from rare variants, whereas traditional twin and family studies are unbiased in this regard ${ }^{24}$. Finally, the diversity of the microbiota residing in the gut might also contribute to the variable response to metformin. For example, metforminassociated change in gut microbiome accounts for a considerable proportion of the difference in taxonomic composition between patients with T2DM and non-diabetic controls $^{29}$. Examining the diversity of this gut microbiome might, therefore, enable the identification of novel targets for the prevention or management of T2DM as the microbiota genome is easier to modify with prebiotics or probiotics compared to the host genome ${ }^{30}$.

Notably, twin and family studies have been used to estimate the heritability of physiological response to antidiabetic agents in participants without T2DM. For example, in a twin-family study of 100 healthy twins and 25 siblings, the heritability of GLP-1 stimulated insulin secretion during hyperglycaemia to be $53 \%{ }^{31}$. In another family study, the heritability of

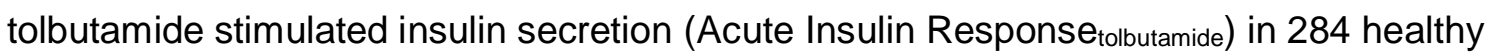
family members of patients with T2DM was estimated to be $69 \%{ }^{32}$. The results of these twin and family studies that include non-diabetic individuals have demonstrated that a large component of the variation in physiological measures in response to antidiabetic drugs is contributed by genetic variants. However, to what extent such high heritability estimates are comparable to that of glycaemic response estimated from population-based studies of patients with T2DM is unclear. Two reasons might account for different heritability estimates between the two study designs. Firstly, twin and family studies are often performed in controlled settings, which have less environmental variance than real world patient populations. Consequently, the same genetic effect sizes could lead to higher heritability estimates. Secondly, the pharmacodynamics in patients with T2DM might differ from that in healthy individuals as the mechanism of glycaemic homeostasis could vary by physiological states in which different functional pathways are involved ${ }^{33}$. 
Most of the robust findings in pharmacogenomic studies to date are related to severe $A D R s^{2}$. The variants associated with these rare ADRs often confer a large risk. For example carrying the HLA-B ${ }^{\star} 57: 01$ allele causes an 80 fold $\left(p=9.0 \times 10^{-9}\right)$ increased risk of flucloxacillin induced liver injury compared to non-carriers of this allele ${ }^{34}$. Encouraged by findings like this, it has been proposed that many of the drug-response variants should have considerable, clinically significant impact on treatment outcome ${ }^{35,36}$. This proposal is supported by the hypothesis that drug response variants lack the evolutionary constraint that has filtered out large disease risk variants via natural selection ${ }^{35}$. However, two explanations exist as to why large impact pharmacogenomics variants are unusual, especially for variants affecting treatment efficacy. Firstly, pharmaceutical interventions often achieve clinical impact via complex metabolic networks, which rely on redundant pathways and synergistic effects to maintain their robustness when confronted with external stimuli ${ }^{37}$. Partial or complete impairment of one node in the network is, therefore, more likely to have marginal impact on the treatment efficacy than a complete shutdown on all the relevant pathways. Secondly, the established spectrum of large impact ADR variants might also reflect a publication bias, which accumulated the so called 'low hanging fruit' that have been identified by pharmacogenomic studies often using fewer than 1000 cases $^{3,38}$. Although the genetic architecture of rare ADRs might be akin to those of polygenic diseases in which large impact variants dominate ${ }^{3}$, we anticipate the genetic architecture of treatment efficacy and mild ADRs would both encompass a spectrum of rare-to-common variants with moderate effect sizes. This notion is in line with the fact that rare variants with moderate impact have been successfully identified for common diseases such as T2DM by sequencing and imputationbased rare variant association studies of over one hundred thousand samples ${ }^{9}$. Assembling large samples would, therefore, enable the identification of more drug response variants by future pharmacogenomic studies.

\section{Pharmacogenomics of T2DM drugs}

Owing to the considerable variability in response to existing drugs to treat diabetes mellitus, a large number of pharmacogenetic studies have been published, but only one pharmacogenomic GWAS study of metformin treatment efficacy reported ${ }^{39}$. These studies each focused on a single oral agent and have been the subject of many previous reviews ${ }^{40-}$ 42. No report exists on the pharmacogenetics of drug-drug interaction, despite a large number of patients requiring multiple agents to combat diabetes progression and maintain glycaemic control. In this section we summarize the replicated findings in studies of treatment efficacy and place more emphasis on the investigation of adverse effects (Table 2). 


\section{Treatment efficacy}

Very few robust pharmacogenetic findings related to treatment efficacy of diabetes mellitus drugs have been reported. Previous candidate gene studies largely focused on drug transporters or metabolizing enzyme variants that have been implicated in the pharmacokinetics of drug exposure ${ }^{41}$. Variation in metformin pharmacokinetics is mainly the result of variants in transporters SLC22A1 (encoding OCT1) and SLC47A1 (encoding MATE1 $)^{43,44}$. However, the most investigated reduced-function OCT1 variants with low transporter activity showed no consistent impact on glycaemic control in patients ${ }^{45-48}$. Sulfonylureas are mainly metabolized by Cytochrome P450 2C9 which is encoded by CYP2C9. Individuals with the loss-of-function variants in CYP2C9 have higher drug exposure, and this in turn does lead to consistent observations of greater glycaemic response than those carrying the wild-type alleles ${ }^{49-51}$.

Studies of potential pharmacodynamic variants, which might affect how antidiabetic agents alter glucose levels, have largely focused on the genes involved in glucose metabolism and the risk of developing T2DM. One replicated finding is seen for TCF7L2 and sulfonylurea response, where the allele associated with reduced $\beta$-cell function and, therefore, increased risk of T2DM is also associated with reduced efficacy of sulfonylureas ${ }^{52-54}$. The $\mathrm{K}$ allele of the E23K variant in KCNJ11, which encodes the known targets of sulfonylureas, also had association with greater glycaemic response in multiple studies ${ }^{55-57}$. These observations of E23K carriers were consistent with the evidence that neonates with monogenic diabetes who have causal variants in $K C N J 11$ could be effectively treated with sulfonylureas ${ }^{18}$. Another replicated finding is the association between PPARG Pro12Ala variant and response to Thiazolidinediones (commonly known as TZDs). A few studies each using less than 200 participants consistently reported the T2DM risk Pro allele is associated with poor glycaemic response ${ }^{58-60}$. For the newer agents such as gliptins and SGLT2 inhibitors, pharmacogenetic studies of treatment efficacy have been sparse with only one relatively large study reporting the association and replication between rs7202877 near CTRB1/2 and response to gliptins ${ }^{61}$.

The only GWAS of any antidiabetic agent published to date identified a variant rs 11212617 near the ATM locus being associated with glycaemic response to metformin ${ }^{39}$. Independent replications were later reported in multiple cohorts of different ancestries, strengthening the evidence that the variant is the most established drug response variant for antidiabetic drug $^{62,63}$. Given the moderate impact on glycaemic response to metformin, this variant is not a useful biomarker that can substantially increase our ability to accurately predict the treatment outcome in individual patients. However, as this variant has no functional link to any known metformin mode-of-action, it demonstrates that such a pharmacogenomic 
discovery could reveal novel mechanisms of action of an antidiabetic drug, which might in turn identify further pathways to target with new therapeutic agents. The finding that variants near ATM are associated with metformin response has prompted further study of the genes at this locus in relation to glucose metabolism and metformin response. For example, in a small study of patients with ataxia telangiectasia who have recessive loss of function mutations in ATM, the investigators identified an impaired glucose tolerance and insulin resistance supporting a potential role for ATM in glucose metabolism and metformin response ${ }^{64}$.

\section{ADRs}

Pharmacogenetic studies have also been performed to help in the understanding of ADRs associated with antidiabetic drugs. Key ADRs studied include sulfonylurea-induced hypoglycaemia, TZD-associated oedema, hepatotoxicity and heart failure, and metformin associated gastrointestinal (Gl) disturbance ${ }^{65-69}$. To date, no studies have been published on the pharmacogenomics of the potentially fatal but rare ADRs of metformin-associated lactic acidosis $^{70}$. Thus far, the investigators in all the published pharmacogenetic studies have adopted a candidate gene approach. For sulfonylurea-induced hypoglycaemia, a number of small studies using no more than 108 patients have been conducted and they consistently showed that the loss-of-function CYP2C9 variants, which are associated with increased drug exposure $^{66}$, are also associated with a higher risk of hypoglycaemia ${ }^{65}$, which is consistent with the efficacy studies showing these variants are associated with greater glucose reduction than wild-type alleles ${ }^{45}$. For TZDs, the results have indicated that variants in GSTT1 and CYP2C19 were associated with troglitazone-induced hepatotoxicity ${ }^{68,71}$; whilst variants in NFATC2 are associated with the rate of rosiglitazone-induced oedema ${ }^{67}$. Although some safety concerns have been raised against the newer agents, such as genital and urinary tract infections associated with SGLT2 inhibitors and the pancreatitis and liver dysfunction associated with gliptins ${ }^{70}$, to our knowledge, no pharmacogenetic reports on these ADRs exist.

Metformin causes gastrointestinal disturbance in as many as $20-40 \%$ of patients with $5-10 \%$ of patients not being able to tolerate this drug $^{70}$. The biological mechanism for gastrointestinal intolerance to metformin remains poorly understood. Based on the hypothesis that individuals who are intolerant of metformin are exposed to higher concentrations of the drug in the gastrointestinal tract, investigators have explored variants in transporter genes such as OCT1 ${ }^{72}$. In a large study of 2,166 patients, both reducedfunction variants in OCT1 and co-medication of certain OCT1 inhibitors (that is, verapamil, proton pump inhibitors, citalopram, codeine and doxazosin) significantly increased the risk of metformin induced gastrointestinal side effect ${ }^{69}$. The combination of carrying the genetic 
variants and taking co-medications could result in a four-fold $(p<0.001)$ increased risk of gastrointestinal side effect ${ }^{69}$. Therefore this large impact on metformin induced gastrointestinal side effects by these OCT1 variants have the potential to be translated into clinical practice by prescreening the patients on OCT1 genotype and co-medication of OCT1 inhibitors. Moreover, this study highlighted two key factors for successful pharmacogenomics of antidiabetic drugs: large sample sizes are required for adequate statistical power; and the need to consider co-prescribed medication that potentially interacts with these agents. This result has since been replicated in an independent study which showed the same genetic variants were associated with increased risk ( $O R=2.3 ; p=0.02)$ of less severe metformin intolerance ${ }^{73}$.

\section{Genetics and drug target validation}

A major challenge in the modern pharmaceutical industry is that fewer than $15 \%$ of drugs entering the development pipeline make it to market $^{74}$, a vastly expensive, inefficient and wasteful process. Toxicity and lack of efficacy both contribute to the high failure rate of new drugs development, which reflects the ineffectiveness of conventional target validation methods used in preclinical studies. Moreover, the model systems used in the preclinical studies often fail to represent the real biological system working in humans.

Genetic variants that arise as a result of historical mutation and recombination events can be viewed as naturally occurring experiments that perturb human gene function. These naturally occurring mutations are an opportunity to see the impact of perturbing a gene (for example, by a potential novel drug) on disease risk and off-target effects without the need to develop and trial the drug. For example, in a large survey of 61,104 drugs across the various stages of development, those drug candidates that targeted proteins encoded by genes with robust human genetic evidence (GWAS evidence or link to Mendelian diseases in OMIM) are twice as likely to be therapeutically valid ${ }^{75}$.

To address the question of how to harness the human genetic evidence to guide target validation in drug development, the 'therapeutic hypothesis' has been proposed ${ }^{76}$. Central to this approach is the concept of a genetic evidence based 'dose-response' curve. An example of translating such a hypothesis into a new drug is the development of the PCSK9 inhibitors to reduce levels of LDL cholesterol ${ }^{77}$. Rare 'gain-of-function' variants in PSCK9 led to high levels of LDL cholesterol and increased the incidence of coronary heart disease risk $^{78}$. Conversely, rare 'loss-of-function' variants resulted in lower LDL cholesterol levels and a reduced risk of coronary heart disease ${ }^{79}$. GWAS also identified common variants in other genes such as SORT1 and LDLR with mild impact on LDL cholesterol levels and risk of coronary heart disease ${ }^{80,81}$. These experiments (which are essentially designed by nature) 
have demonstrated that reducing levels of LDL cholesterol and the risk of coronary heart disease is possible by inhibiting the function of PCSK9 without any observable adverse effects ${ }^{77}$. With further support by other mechanistic evidence derived from model systems, a new generation of LDL cholesterol lowering PCSK9 inhibitors have been developed, tested and licensed for use ${ }^{82}$.

Dozens of common and rare variants have been convincingly associated with glycaemic control and the risk of developing $\mathrm{T}^{2} \mathrm{DM}^{10}$. Representatives from both academia and pharmaceutical companies have now formed the Accelerating Medicines Partnership to enhance the translation of human genomic research outputs into the development of new drugs (https://www.nih.gov/research-training/accelerating-medicines-partnership-amp) ${ }^{83}$. As one of the three prioritized area, the Accelerating Medicines Partnership aims to establish an open-access portal for T2DM genetics research (http://www.type2diabetesgenetics.org), which will pool genomic and phenotyping data to facilitate novel data mining efforts ${ }^{83}$. One target of potential interest raised by the Accelerating Medicines Partnership is the zinctransporter-encoding SLC30A8. The use of GWAS has established robust evidence that the common coding variant Arg325Trp is associated with the risk of T2DM ${ }^{84}$. The results of mechanistic studies in humans and mice have both indicated that the reduced zinc transporter activity allele is associated with an increased risk of T2DM ${ }^{85,86}$ However, in a sequencing based study of $\sim 150,000$ individuals, rare protein-truncating variants In SLC30A8 protected the carriers from developing T2DM ${ }^{9}$. This conflicting genetic evidence does not provide a consistent dose-response curve based on the functional characterization of the variants ${ }^{87}$. Further mechanistic studies, especially those involving intensive phenotyping of individuals carrying rare extreme functional SLC30A8 variants will be useful to validate whether inhibiting or enhancing the zinc transporter 8 function could be the therapeutic option for treating T2DM. The example provided by SLC30A8 demonstrates that human genetic evidence is not always sufficient to validate a gene/protein as a drug target, especially in situations when uncertainty surrounds the exact mechanism of how target genes alter a phenotype of interest.

The discovery of sodium glucose transporter 2 (SGLT2) inhibitors to treat T2DM is another example of how genetic evidence can be used in assisting drug development (Figure 2). Early evidence indicated that phlorizin, a natural inhibitor of SGLT2 and isolated from the bark of apple trees, restored euglycaemia and insulin sensitivity in animal models of T2DM ${ }^{88}$. After the cloning of SLC5A2 which encodes SGLT2, functional variants in SGLT2 have been linked to familial renal glycosuria (http://www.omim.org/entry/233100) ${ }^{89}$. Patients with familial renal glycosuria, who carry a spectrum of $>50$ mild heterozygote to severe homozygote SGLT2 loss-of-function variants, have different levels of glycosuria but apparently normal 
renal functions, normal glucose concentrations and general health ${ }^{90}$. Here the genetic evidence not only validated the therapeutic potential of inhibiting SGLT2 but also provided critical evidence that selective inhibitors targeting the protein would result in no long-term ADRs. Consequently, several selective SGLT2 inhibitors have been successfully developed and licensed to treat T2DM ${ }^{14}$. Aside from their glucose lowering effect, these SGLT2 inhibitors have an average impact of $1.63 \mathrm{~kg}(p<0.001)$ weight reduction benefit ${ }^{16,91}$, which is increasingly being considered as an important component in the management of T2DM ${ }^{92}$.

\section{Pharmacogenomics and drug discovery}

Conventional pharmacogenetics, which is based on candidate genes has been used in all stages of the drug development pipeline, from target identification, to clinical trials and postmarket analysis of $\mathrm{ADRs}^{35}$. As pharmacogenetics moves to pharmacogenomics, this genome-wide hypothesis free approach could also have more applications in drug discovery and development ${ }^{20}$.

The proposed 'therapeutic hypothesis' is based on the principle that human genetics is an experiment of nature, which shows what phenotypic outcomes the natural perturbations of gene functions can lead to in a human population ${ }^{76}$. Clearly human genetics has the advantage that the experimental system is in living humans as opposed to the cell or animal model systems used in preclinical studies. However, the ideal system for validating a therapeutic hypothesis would be a genetic study carried out in the exact context the new drug is developed for. Genetic evidence of disease risk could be useful in validating the targets for a preventive drug but less informative to predict the therapeutic potential for disease management. For example, let us consider using human genetic evidence to validate candidate drug targets to improve glycaemic control in patients with T2DM. The most available candidates would be those genes with established variants associated with the risk of developing T2DM or poor glycaemic control in the general population. However, it has been suggested that the mechanism regulating glycaemia can vary between different physiological states ${ }^{33}$. The variants affecting glycaemia in normal individuals and those associated with T2DM risk only partially overlap ${ }^{33}$, suggesting perturbations of glycaemia are not always linked to the risk of T2DM. Similarly, the variants conferring risk to T2DM have little impact on the rate of disease progression ${ }^{93}$, which suggests that the mechanism controlling glycaemia when T2DM has developed might be different from those involved in its development. Consequently, targeting the genes involved in regulation of glycaemia in healthy individuals or the risk of T2DM might not have the desired glycaemic impact on the management of T2DM, especially if their biological functions have been altered by the onset of the disease. In the context of disease management, pharmacogenomic studies of existing 
antidiabetic drugs can identify genes involved in glycaemic control in patients with T2DM. Robust pharmacogenomic findings would, therefore, provide physiological state specific information for drug target validation in addition to other evidence of normal glycaemic control and risk of T2DM derived from human genetic studies.

Table 1 outlines the differences between pharmacogenomic studies of drugs to treat T2DM and genomics of other diabetes mellitus-related traits. Compared to the wealth of findings from other areas of human genetics, to date pharmacogenomics has yielded less robust results, in part owing to the challenge to assemble tens of thousands of samples that could provide adequate statistical power to detect variants with moderate effect. However, we anticipate future drug discovery and drug development to benefit more from the pharmacogenomic findings derived from larger samples that are increasingly available from biobanks linked to electronic health record data (e.g. the UKBiobank). Notably, longitudinal data from clinical trials or health record linkage do enable the study of outcomes other than simple measures of glycaemia. For example, given a sufficient sample size, using pharmacogenomics to assess cardiovascular endpoints of antidiabetic drugs might be possible. In addition, large samples would also enable pharmacogenomics to joint analyse multiple outcomes (for example both glycaemic benefit and weight benefit of metformin), which would help to identify variants associated with pleiotropic effects of antidiabetic drugs.

Avoiding ADRs or off-target effects is also an important consideration in drug development. The very existence of ADRs is an illustration of our incomplete understanding of the complex interactions between our biological system and any designed interventions ${ }^{94}$. The increased availability of genome-wide screening tools will enable the identification of more ADR variants at an early stage of drug development, which has a number of clear advantages. For example, if the ADR variants were identified before phase 3 clinical trials, recruitment by genotype trials could be performed to evaluate the efficacy and risk in stratified patient subgroups ${ }^{35}$. When a drug is approved for marketing, understanding the genetic basis of severe ADR could also help the continuous development of the drug by prescreening the genotype ahead of treatment ${ }^{95}$.

\section{A system biology view of drug response}

One of the biggest challenges facing contemporary biological research is to understand the complex biological networks that function in a living system ${ }^{96}$. This complexity can be seen in T2DM, where the genes harbouring the established aetiological variants have an excess of interactions within a high-confidence interaction network ${ }^{97}$. The variants in this network affect the protein function, stability of protein or transcript, and expression of individual genes, which collectively perturb or rewire the network structure to alter the risk of T2DM ${ }^{98,99}$. 
Interestingly, when considering a network of disease genes and genes encoding known drug targets, the distance between genes reported in GWAS and known drug targets are shorter than that between random gene-drug targets pairs ${ }^{100}$. This is predominantly driven by a three-fold enrichment of drug target genes among the first neighbours of the GWAS reported genes. More evidence of direct overlap between the drug target genes and disease risk genes was also presented in a gene set enrichment analysis ${ }^{101}$. In this study a set of 102 target genes for existing diabetes mellitus drugs (e.g. insulin, metformin and TZDs), as curated from the literature, showed significant enrichment of genetic association with T2DM susceptibility ${ }^{101}$. Such findings indicate that potential drug targets are enriched in a disease network, but might only be identified when considering the network as a whole, rather than individual disease genes.

When modelling a drug intervention in a functional network, it is important to consider how the drug alters the functional network at the levels of cell, tissue and disease state, which will ultimately determine the beneficial and harmful effects of the drug. So far our knowledge of biological networks has been largely limited to the generic, static models lacking such contextual information ${ }^{102}$. Research in system biology is beginning to offer more comprehensive cell lineage and tissue specific networks ${ }^{103-105}$, which will enable the modelling of drug intervention in more specific contexts such as the cell type, tissue and the physiological state. Such analyses will provide insights into how drugs can achieve the desired therapeutic effect in target tissue or known site of action, but importantly highlight the potential undesirable off-target effects in other contexts. Moreover, adopting a system-wide approach might change the focus of drug development for complex diseases such as T2DM from targeting an individual protein or gene to system-wide attacks on multiple dynamic targets $^{106}$.

\section{Conclusions}

The availability of affordable high-throughput genomic technologies has expanded our knowledge about the multifactorial aetiology of T2DM. Studies adopting such genome-wide approaches to investigate the response of existing antidiabetic drugs have been limited, but have the potential to improve our understanding of the biological mechanisms underlying treatment efficacy and side effects. With the major investments in precision medicine in the $U^{107}$, the 100,000 genomes project in the $\mathrm{UK}^{108}$, and the EU funded stratified medicine in diabetes mellitus initiative IMI-DIRECT ${ }^{109}$ (Innovative Medicines Initiative: Dlabetes REsearCh on patient straTification), more findings from adequately powered pharmacogenomic studies are expected to complement other human genetic discoveries to facilitate more efficient antidiabetic drug discovery programs. 


\section{References}

1. Visscher, P.M., Brown, M.A., McCarthy, M.I. \& Yang, J. Five years of GWAS discovery. Am J Hum Genet 90, 7-24 (2012).

2. Daly, A.K. Pharmacogenomics of adverse drug reactions. Genome Med 5, 5 (2013).

3. Zhou, K. \& Pearson, E.R. Insights from genome-wide association studies of drug response. Annu Rev Pharmacol Toxicol 53, 299-310 (2013).

4. American Diabetes, A. Diagnosis and classification of diabetes mellitus. Diabetes Care $\mathbf{2 7}$ Suppl 1, S5-S10 (2004).

5. Stumvoll, M., Goldstein, B.J. \& van Haeften, T.W. Type 2 diabetes: principles of pathogenesis and therapy. Lancet 365, 1333-46 (2005).

6. Morris, A.P. et al. Large-scale association analysis provides insights into the genetic architecture and pathophysiology of type 2 diabetes. Nat Genet 44, 981-90 (2012).

7. Prasad, R.B. \& Groop, L. Genetics of type 2 diabetes-pitfalls and possibilities. Genes (Basel) 6, 87-123 (2015).

8. Steinthorsdottir, V. et al. Identification of low-frequency and rare sequence variants associated with elevated or reduced risk of type 2 diabetes. Nat Genet 46, 294-8 (2014).

9. Flannick, J. et al. Loss-of-function mutations in SLC30A8 protect against type 2 diabetes. Nat Genet 46, 357-63 (2014).

10. Mohlke, K.L. \& Boehnke, M. Recent advances in understanding the genetic architecture of type 2 diabetes. Hum Mol Genet 24, R85-92 (2015).

11. Holman, R.R., Paul, S.K., Bethel, M.A., Matthews, D.R. \& Neil, H.A. 10-year follow-up of intensive glucose control in type 2 diabetes. N Engl J Med 359, 1577-89 (2008).

12. Stratton, I.M. et al. Association of glycaemia with macrovascular and microvascular complications of type 2 diabetes (UKPDS 35): prospective observational study. BMJ 321, 405-12 (2000).

13. Intensive blood-glucose control with sulphonylureas or insulin compared with conventional treatment and risk of complications in patients with type 2 diabetes (UKPDS 33). UK Prospective Diabetes Study (UKPDS) Group. Lancet 352, 837-53 (1998).

14. Bailey, C.J. The Current Drug Treatment Landscape for Diabetes and Perspectives for the Future. Clin Pharmacol Ther 98, 170-84 (2015).

15. Deacon, C.F. Dipeptidyl peptidase-4 inhibitors in the treatment of type 2 diabetes: a comparative review. Diabetes Obes Metab 13, 7-18 (2011).

16. Tahrani, A.A., Barnett, A.H. \& Bailey, C.J. SGLT inhibitors in management of diabetes. Lancet Diabetes Endocrinol 1, 140-51 (2013).

17. Pearson, E.R. et al. Genetic cause of hyperglycaemia and response to treatment in diabetes. Lancet 362, 1275-81 (2003). 
18. Gloyn, A.L. et al. Activating mutations in the gene encoding the ATP-sensitive potassiumchannel subunit Kir6.2 and permanent neonatal diabetes. N Engl J Med 350, 1838-49 (2004).

19. Carr, D.F., Alfirevic, A. \& Pirmohamed, M. Pharmacogenomics: Current State-of-the-Art. Genes (Basel) 5, 430-43 (2014).

20. Goldstein, D.B., Tate, S.K. \& Sisodiya, S.M. Pharmacogenetics goes genomic. Nat Rev Genet 4, 937-47 (2003).

21. Goldstein, D.B. et al. Sequencing studies in human genetics: design and interpretation. Nat Rev Genet 14, 460-70 (2013).

22. Mizzi, C. et al. Personalized pharmacogenomics profiling using whole-genome sequencing. Pharmacogenomics 15, 1223-34 (2014).

23. Zhou, K. et al. Heritability of variation in glycaemic response to metformin: a genome-wide complex trait analysis. Lancet Diabetes Endocrinol 2, 481-7 (2014).

24. Yang, J. et al. Genome partitioning of genetic variation for complex traits using common SNPs. Nat Genet 43, 519-25 (2011).

25. Visscher, P.M. et al. Statistical power to detect genetic (co)variance of complex traits using SNP data in unrelated samples. PLoS Genet 10, e1004269 (2014).

26. Yang, J. et al. Genetic variance estimation with imputed variants finds negligible missing heritability for human height and body mass index. Nat Genet 47, 1114-20 (2015).

27. Elks, C.E. et al. Variability in the heritability of body mass index: a systematic review and meta-regression. Front Endocrinol (Lausanne) 3, 29 (2012).

28. Yang, J. et al. Common SNPs explain a large proportion of the heritability for human height. Nat Genet 42, 565-9 (2010).

29. Forslund, K. et al. Disentangling type 2 diabetes and metformin treatment signatures in the human gut microbiota. Nature 528, 262-6 (2015).

30. McCreight, L.J., Bailey, C.J. \& Pearson, E.R. Metformin and the gastrointestinal tract. Diabetologia (2016).

31. Simonis-Bik, A.M. et al. Genetic influences on the insulin response of the beta cell to different secretagogues. Diabetologia 52, 2570-7 (2009).

32. Gjesing, A.P. et al. High heritability and genetic correlation of intravenous glucose- and tolbutamide-induced insulin secretion among non-diabetic family members of type 2 diabetic patients. Diabetologia 57, 1173-81 (2014).

33. De Silva, N.M. \& Frayling, T.M. Novel biological insights emerging from genetic studies of type 2 diabetes and related metabolic traits. Curr Opin Lipido/ 21, 44-50 (2010).

34. Daly, A.K. et al. HLA-B*5701 genotype is a major determinant of drug-induced liver injury due to flucloxacillin. Nat Genet 41, 816-9 (2009).

35. Harper, A.R. \& Topol, E.J. Pharmacogenomics in clinical practice and drug development. Nat Biotechnol 30, 1117-24 (2012). 
36. Maranville, J.C. \& Cox, N.J. Pharmacogenomic variants have larger effect sizes than genetic variants associated with other dichotomous complex traits. Pharmacogenomics J (2015).

37. Hellerstein, M.K. Exploiting complexity and the robustness of network architecture for drug discovery. J Pharmacol Exp Ther 325, 1-9 (2008).

38. Nelson, M.R. et al. Genome-wide approaches to identify pharmacogenetic contributions to adverse drug reactions. Pharmacogenomics J 9, 23-33 (2009).

39. Zhou, K. et al. Common variants near ATM are associated with glycemic response to metformin in type 2 diabetes. Nat Genet 43, 117-20 (2011).

40. Becker, M.L., Pearson, E.R. \& Tkac, I. Pharmacogenetics of Oral Antidiabetic Drugs. Int J Endocrinol 2013, 686315 (2013).

41. Semiz, S., Dujic, T. \& Causevic, A. Pharmacogenetics and personalized treatment of type 2 diabetes. Biochem Med (Zagreb) 23, 154-71 (2013).

42. Todd, J.N. \& Florez, J.C. An update on the pharmacogenomics of metformin: progress, problems and potential. Pharmacogenomics 15, 529-39 (2014).

43. Shu, Y. et al. Effect of genetic variation in the organic cation transporter 1, OCT1, on metformin pharmacokinetics. Clin Pharmacol Ther 83, 273-80 (2008).

44. Graham, G.G. et al. Clinical pharmacokinetics of metformin. Clin Pharmacokinet 50, 81-98 (2011).

45. Zhou, K. et al. Reduced-function SLC22A1 polymorphisms encoding organic cation transporter 1 and glycemic response to metformin: a GoDARTS study. Diabetes 58, 1434-9 (2009).

46. Jablonski, K.A. et al. Common variants in $\mathbf{4 0}$ genes assessed for diabetes incidence and response to metformin and lifestyle intervention in the diabetes prevention program. Diabetes 59, 2672-81 (2010).

47. Becker, M.L. et al. Genetic variation in the organic cation transporter 1 is associated with metformin response in patients with diabetes mellitus. Pharmacogenomics J 9, 242-7 (2009).

48. Shu, Y. et al. Effect of genetic variation in the organic cation transporter 1 (OCT1) on metformin action. J Clin Invest 117, 1422-31 (2007).

49. Zhou, K. et al. Loss-of-function CYP2C9 variants improve therapeutic response to sulfonylureas in type 2 diabetes: a Go-DARTS study. Clin Pharmacol Ther 87, 52-6 (2010).

50. Becker, M.L. et al. Cytochrome P450 $2 \mathrm{Cg} * 2$ and *3 polymorphisms and the dose and effect of sulfonylurea in type II diabetes mellitus. Clin Pharmacol Ther 83, 288-92 (2008).

51. Suzuki, K. et al. Effect of CYP2C9 genetic polymorphisms on the efficacy and pharmacokinetics of glimepiride in subjects with type 2 diabetes. Diabetes Res Clin Pract 72, 148-54 (2006).

52. Pearson, E.R. et al. Variation in TCF7L2 influences therapeutic response to sulfonylureas: a GoDARTs study. Diabetes 56, 2178-82 (2007).

53. Schroner, Z. et al. Effect of sulphonylurea treatment on glycaemic control is related to TCF7L2 genotype in patients with type 2 diabetes. Diabetes Obes Metab 13, $89-91$ (2011). 
54. Javorsky, M. et al. Association between TCF7L2 Genotype and Glycemic Control in Diabetic Patients Treated with Gliclazide. Int J Endocrinol 2013, 374858 (2013).

55. Javorsky, M. et al. KCNJ11 gene E23K variant and therapeutic response to sulfonylureas. Eur J Intern Med 23, 245-9 (2012).

56. Feng, Y. et al. Ser1369Ala variant in sulfonylurea receptor gene ABCC8 is associated with antidiabetic efficacy of gliclazide in Chinese type 2 diabetic patients. Diabetes Care 31, 1939-44 (2008).

57. Zhang, H., Liu, X., Kuang, H., Yi, R. \& Xing, H. Association of sulfonylurea receptor 1 genotype with therapeutic response to gliclazide in type 2 diabetes. Diabetes Res Clin Pract 77, 58-61 (2007).

58. Pei, Q. et al. PPAR-gamma2 and PTPRD gene polymorphisms influence type 2 diabetes patients' response to pioglitazone in China. Acta Pharmacol Sin 34, 255-61 (2013).

59. Kang, E.S. et al. Effects of Pro12Ala polymorphism of peroxisome proliferator-activated receptor gamma2 gene on rosiglitazone response in type 2 diabetes. Clin Pharmacol Ther 78, 202-8 (2005).

60. Hsieh, M.C. et al. Common polymorphisms of the peroxisome proliferator-activated receptor-gamma (Pro12Ala) and peroxisome proliferator-activated receptor-gamma coactivator-1 (Gly482Ser) and the response to pioglitazone in Chinese patients with type 2 diabetes mellitus. Metabolism 59, 1139-44 (2010).

61. $\mathrm{t}$ Hart, L.M. et al. The CTRB1/2 locus affects diabetes susceptibility and treatment via the incretin pathway. Diabetes 62, 3275-81 (2013).

62. van Leeuwen, N. et al. A gene variant near ATM is significantly associated with metformin treatment response in type 2 diabetes: a replication and meta-analysis of five cohorts. Diabetologia 55, 1971-7 (2012).

63. Zhou, Y. et al. RS11212617 is associated with metformin treatment response in type 2 diabetes in Shanghai local Chinese population. Int J Clin Pract 68, 1462-6 (2014).

64. Connelly, P.J. et al. Recessive mutations in the cancer gene Ataxia Telangiectasia Mutated (ATM), at a locus previously associated with metformin response, cause dysglycaemia and insulin resistance. Diabetic Medicine (In press).

65. Gokalp, O. et al. Mild hypoglycaemic attacks induced by sulphonylureas related to CYP2C9, CYP2C19 and CYP2C8 polymorphisms in routine clinical setting. Eur J Clin Pharmacol 67, 1223-9 (2011).

66. Zhang, Y. et al. Influence of CYP2C9 and CYP2C19 genetic polymorphisms on pharmacokinetics of gliclazide MR in Chinese subjects. Br J Clin Pharmacol 64, 67-74 (2007).

67. Bailey, S.D. et al. Variation at the NFATC2 locus increases the risk of thiazolidinedioneinduced edema in the Diabetes REduction Assessment with ramipril and rosiglitazone Medication (DREAM) study. Diabetes Care 33, 2250-3 (2010).

68. Watanabe, I. et al. A study to survey susceptible genetic factors responsible for troglitazoneassociated hepatotoxicity in Japanese patients with type 2 diabetes mellitus. Clin Pharmacol Ther 73, 435-55 (2003). 
69. Dujic, T. et al. Association of Organic Cation Transporter 1 With Intolerance to Metformin in Type 2 Diabetes: A GoDARTS Study. Diabetes 64, 1786-93 (2015).

70. Bailey, C.J. Safety of antidiabetes medications: An update. Clin Pharmacol Ther 98, 185-95 (2015).

71. Kumashiro, R. et al. Association of troglitazone-induced liver injury with mutation of the cytochrome P450 2C19 gene. Hepatol Res 26, 337-342 (2003).

72. Tarasova, L. et al. Association of genetic variation in the organic cation transporters OCT1, OCT2 and multidrug and toxin extrusion 1 transporter protein genes with the gastrointestinal side effects and lower BMI in metformin-treated type 2 diabetes patients. Pharmacogenet Genomics 22, 659-66 (2012).

73. Dujic, T. et al. Organic Cation Transporter 1 Variants and Gastrointestinal Side Effects of Metformin in Patients with Type 2 Diabetes. Diabetic Medicine (in press).

74. Hay, M., Thomas, D.W., Craighead, J.L., Economides, C. \& Rosenthal, J. Clinical development success rates for investigational drugs. Nat Biotechnol 32, 40-51 (2014).

75. Nelson, M.R. et al. The support of human genetic evidence for approved drug indications. Nat Genet 47, 856-60 (2015).

76. Plenge, R.M., Scolnick, E.M. \& Altshuler, D. Validating therapeutic targets through human genetics. Nat Rev Drug Discov 12, 581-94 (2013).

77. Cohen, J.C. Emerging LDL therapies: Using human genetics to discover new therapeutic targets for plasma lipids. J Clin Lipidol 7, S1-5 (2013).

78. Abifadel, M. et al. Mutations in PCSK9 cause autosomal dominant hypercholesterolemia. Nat Genet 34, 154-6 (2003).

79. Cohen, J.C., Boerwinkle, E., Mosley, T.H., Jr. \& Hobbs, H.H. Sequence variations in PCSK9, low LDL, and protection against coronary heart disease. N Engl J Med 354, 1264-72 (2006).

80. Global Lipids Genetics, C. et al. Discovery and refinement of loci associated with lipid levels. Nat Genet 45, 1274-83 (2013).

81. Schunkert, H. et al. Large-scale association analysis identifies 13 new susceptibility loci for coronary artery disease. Nat Genet 43, 333-8 (2011).

82. Stein, E.A. et al. Effect of a monoclonal antibody to PCSK9 on LDL cholesterol. N Engl J Med 366, 1108-18 (2012).

83. Mullard, A. Drug makers and NIH team up to find and validate targets. Nat Rev Drug Discov 13, 241-3 (2014).

84. Lyssenko, V. et al. Clinical risk factors, DNA variants, and the development of type 2 diabetes. N EnglJ Med 359, 2220-32 (2008).

85. Tamaki, M. et al. The diabetes-susceptible gene SLC30A8/ZnT8 regulates hepatic insulin clearance. J Clin Invest 123, 4513-24 (2013). 
86. Nicolson, T.J. et al. Insulin storage and glucose homeostasis in mice null for the granule zinc transporter ZnT8 and studies of the type 2 diabetes-associated variants. Diabetes 58, 2070-83 (2009).

87. Pearson, E. Zinc transport and diabetes risk. Nat Genet 46, 323-4 (2014).

88. Rossetti, L., Smith, D., Shulman, G.I., Papachristou, D. \& DeFronzo, R.A. Correction of hyperglycemia with phlorizin normalizes tissue sensitivity to insulin in diabetic rats. J Clin Invest 79, 1510-5 (1987).

89. Santer, R. \& Calado, J. Familial renal glucosuria and SGLT2: from a mendelian trait to a therapeutic target. Clin J Am Soc Nephrol 5, 133-41 (2010).

90. Sabino-Silva, R. et al. The $\mathrm{Na}(+) / g l u c o s e$ cotransporters: from genes to therapy. Braz J Med Biol Res 43, 1019-26 (2010).

91. Zhang, M. et al. Dapagliflozin treatment for type 2 diabetes: a systematic review and metaanalysis of randomized controlled trials. Diabetes Metab Res Rev 30, 204-21 (2014).

92. Van Gaal, L. \& Scheen, A. Weight management in type 2 diabetes: current and emerging approaches to treatment. Diabetes Care 38, 1161-72 (2015).

93. Zhou, K. et al. Clinical and genetic determinants of progression of type 2 diabetes: a DIRECT study. Diabetes Care 37, 718-24 (2014).

94. Chang, R.L., Xie, L., Xie, L., Bourne, P.E. \& Palsson, B.O. Drug off-target effects predicted using structural analysis in the context of a metabolic network model. PLoS Comput Biol 6, e1000938 (2010).

95. Mallal, S. et al. HLA-B*5701 screening for hypersensitivity to abacavir. N Engl J Med 358, 568-79 (2008).

96. Csermely, P., Korcsmaros, T., Kiss, H.J., London, G. \& Nussinov, R. Structure and dynamics of molecular networks: a novel paradigm of drug discovery: a comprehensive review. Pharmacol Ther 138, 333-408 (2013).

97. Taneera, J. et al. A systems genetics approach identifies genes and pathways for type 2 diabetes in human islets. Cell Metab 16, 122-34 (2012).

98. Gaulton, K.J. et al. Genetic fine mapping and genomic annotation defines causal mechanisms at type 2 diabetes susceptibility loci. Nat Genet 47, 1415-25 (2015).

99. Carter, H., Hofree, M. \& Ideker, T. Genotype to phenotype via network analysis. Curr Opin Genet Dev 23, 611-21 (2013).

100. Cao, C. \& Moult, J. GWAS and drug targets. BMC Genomics 15 Suppl 4, S5 (2014).

101. Segre, A.V., Wei, N., Altshuler, D. \& Florez, J.C. Pathways targeted by antidiabetes drugs are enriched for multiple genes associated with type 2 diabetes risk. Diabetes 64, 1470-83 (2015).

102. Ideker, T. \& Krogan, N.J. Differential network biology. Mol Syst Biol 8, 565 (2012).

103. Greene, C.S. et al. Understanding multicellular function and disease with human tissuespecific networks. Nat Genet 47, 569-76 (2015). 
104. Bossi, A. \& Lehner, B. Tissue specificity and the human protein interaction network. Mol Syst Biol 5, 260 (2009).

105. Magger, O., Waldman, Y.Y., Ruppin, E. \& Sharan, R. Enhancing the prioritization of diseasecausing genes through tissue specific protein interaction networks. PLoS Comput Biol 8, e1002690 (2012).

106. Erler, J.T. \& Linding, R. Network medicine strikes a blow against breast cancer. Cell 149, 7313 (2012).

107. Collins, F.S. \& Varmus, H. A new initiative on precision medicine. N Eng/ J Med 372, 793-5 (2015).

108. Siva, N. UK gears up to decode 100,000 genomes from NHS patients. Lancet $385,103-4$ (2015).

109. Koivula, R.W. et al. Discovery of biomarkers for glycaemic deterioration before and after the onset of type 2 diabetes: rationale and design of the epidemiological studies within the IMI DIRECT Consortium. Diabetologia 57, 1132-42 (2014).

110. Miller, R.A. et al. Biguanides suppress hepatic glucagon signalling by decreasing production of cyclic AMP. Nature 494, 256-60 (2013).

111. Mulherin, A.J. et al. Mechanisms underlying metformin-induced secretion of glucagon-like peptide-1 from the intestinal L cell. Endocrinology 152, 4610-9 (2011).

112. Gribble, F.M. \& Reimann, F. Pharmacological modulation of K(ATP) channels. Biochem Soc Trans 30, 333-9 (2002).

113. Dornhorst, A. Insulinotropic meglitinide analogues. Lancet 358, 1709-16 (2001).

114. Yki-Jarvinen, H. Thiazolidinediones. N Engl J Med 351, 1106-18 (2004).

115. Verspohl, E.J. Novel therapeutics for type 2 diabetes: incretin hormone mimetics (glucagonlike peptide-1 receptor agonists) and dipeptidyl peptidase-4 inhibitors. Pharmacol Ther 124, 113-38 (2009).

116. Bailey, C.J. Renal glucose reabsorption inhibitors to treat diabetes. Trends Pharmacol Sci 32, 63-71 (2011). 


\section{Key points}

- The list of known variants affecting type 2 diabetes mellitus (T2DM) risk confirms that this disease has a multifactorial aetiology

- The concept of precision medicine has been exemplified in pharmacogenetic studies of monogenic diabetes

- The genetic architecture of mild adverse drug reactions and treatment efficacy for antidiabetic agents probably resembles that of T2DM and other complex traits

- Existing pharmacogenetic evidence of T2DM is limited; future pharmacogenomics studies utilizing large samples will help identify variants that reveal novel mechanism of drug action

- Genetic evidence-based 'dose-response' curves have been used in validating candidate drug targets

- Pharmacogenomic studies adopting a system biology approach are expected to provide context specific evidence for future T2DM drug development

\section{Acknowledgements}

ERP holds a Wellcome Trust New Investigator Award 102820/Z/13/Z. The authors wish to thank Kristoffer Forslund for critical input.

\section{Competing interests statement}

The authors declare no competing interests.

\section{Contributions}

All authors contributed to all aspects of the manuscript. 
Figure 1 | Target organs and action mechanism of antidiabetic drugs. The mechanism for metformin action remains uncertain: metformin might target the liver to reduce gluconeogenesis and skeletal muscles to enhance peripheral glucose utilization ${ }^{110}$ with a possible role in the gut to increase levels of GLP- $1^{111}$. Sulfonylureas and meglitinides increase insulin secretion in the pancreas ${ }^{112,113}$. Thiazolidinediones (TZDs) act as insulin sensitizers in the skeletal muscle, adipose tissue and the liver ${ }^{114}$. GLP-1 agonists target the pancreas to increase insulin secretion and reduce glucagon production, as well as act in the gut to reduce gastric emptying ${ }^{115}$. DPP-4 inhibitors increase the level of endogenous incretin levels by blocking the action of a catalytic enzyme DPP- $4{ }^{115}$. SGLT-2 inhibitors reduce renal glucose reabsorption ${ }^{116}$. Abbreviations: DDP4, dipeptidyl peptidase 4, GLP1RA, glucagon like peptide 1 receptor agonist, SGLT2I, sodium glucose transporter 2 inhibitor

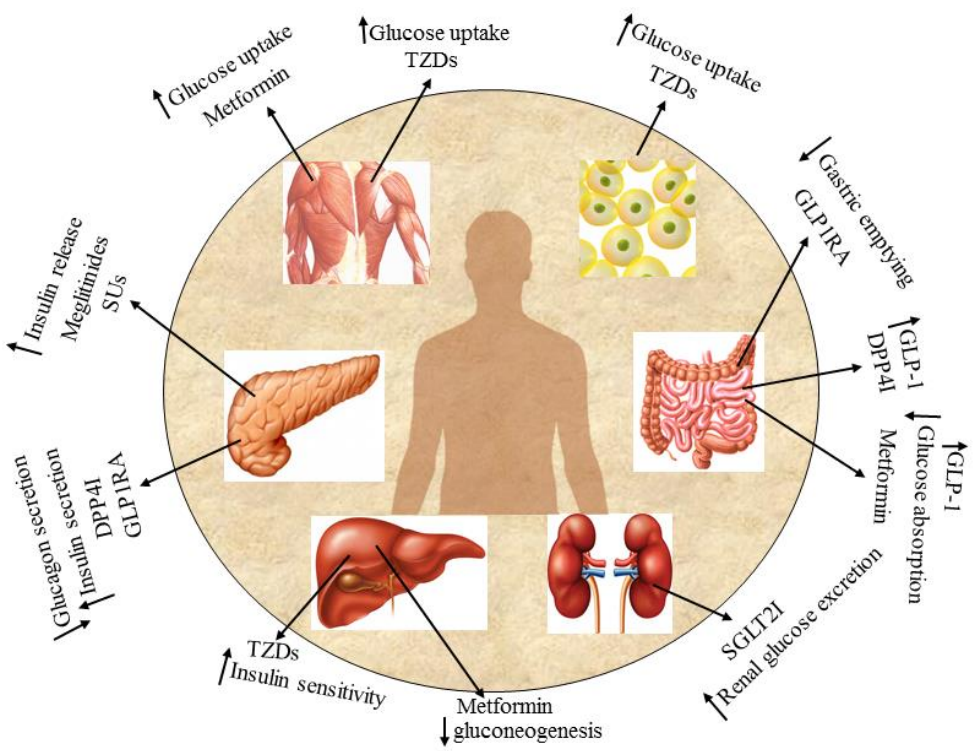


Figure 2 | Dose-response curve for the therapeutic hypothesis of selective SGLT2

inhibitors. The $Y$ axis represent the range of glucose level, in which the high range represents the hyperglycaemic state seen in T2DM as compared to the normal range seen in healthy individuals or those patients with familial renal glycosuria. The $\mathrm{X}$ axis represents a spectrum of naturally occurring SGLT2 loss-of-function variants observed in patients with familial renal glycosuria. The variants were ordered from the mild heterozygotes to the severe homozygotes as defined by the resulting severity of glycosuria. For on-target adverse reactions, the benign glucosuria and apparently normal health seen in these patients also supports the safety profile of selectively inhibiting SGLT2 function in a wide dose window. Abbreviation: SGLT2, sodium glucose transporter 2 .

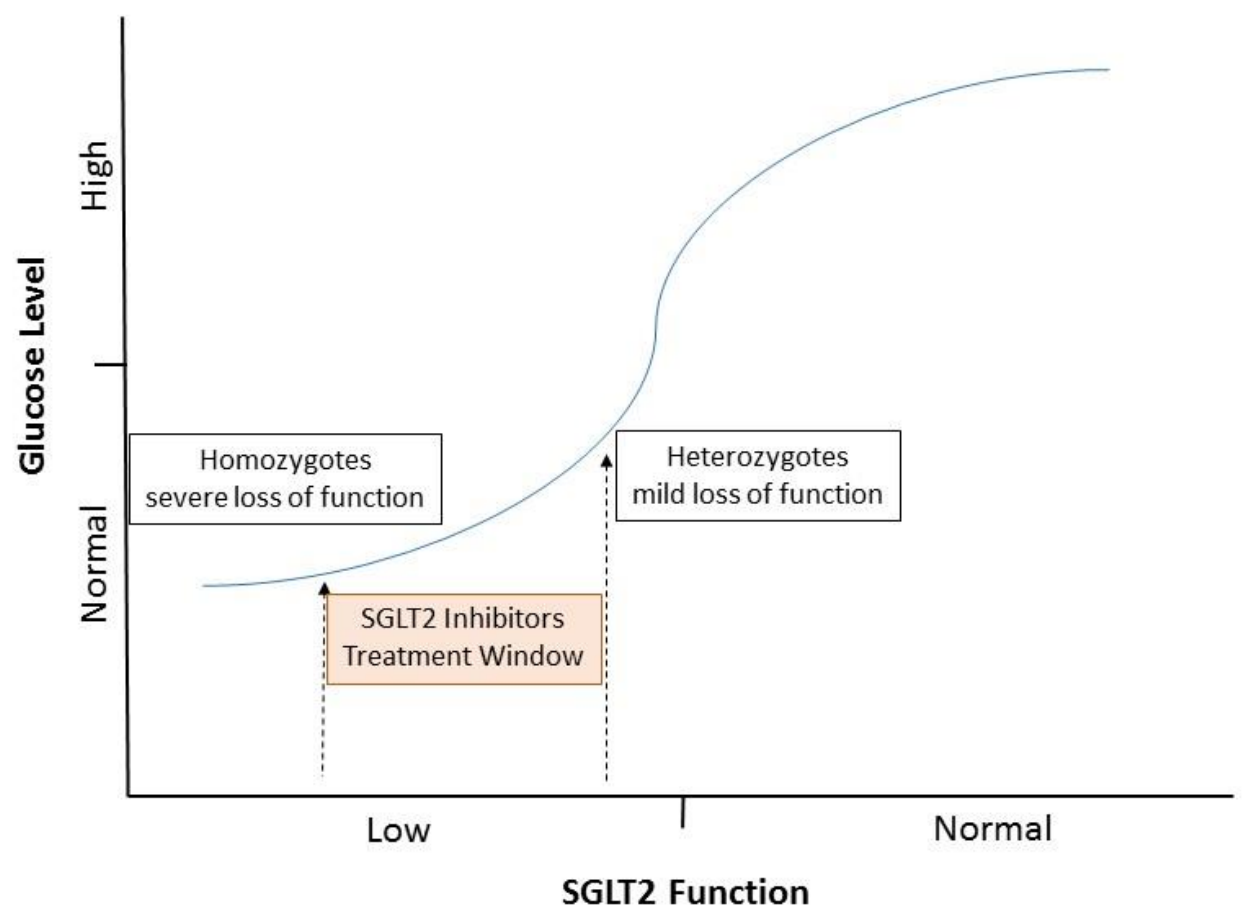




\begin{tabular}{|c|c|c|}
\hline & $\begin{array}{l}\text { Pharmacogenomics of antidiabetic } \\
\text { drugs }\end{array}$ & $\begin{array}{l}\text { Genomic studies of other diabetes } \\
\text { mellitus-related traits }\end{array}$ \\
\hline Sample sizes & Currently $<10,000$ individuals & Currently $>100,000$ individuals \\
\hline $\begin{array}{l}\text { Number of } \\
\text { established } \\
\text { variants }\end{array}$ & Only a few & $\begin{array}{l}>120 \text { for T2DM risk } \\
>83 \text { for other diabetes mellitus-related } \\
\text { traits }\end{array}$ \\
\hline Data type & Longitudinal & Cross sectional \\
\hline Outcomes & $\begin{array}{l}\text { Physiological response e.g. } \\
\text { insulin secretion } \\
\text { Treatment efficacy: glucose } \\
\text { reduction } \\
\text { Hard endpoints: cardiovascular } \\
\text { risk } \\
\text { Other outcomes: weight } \\
\text { reduction }\end{array}$ & $\begin{array}{l}\text { Onset of type } 2 \text { diabetes } \\
\text { Hyperglycaemia: fasting glucose level, } \\
\text { HbA1c levels } \\
\text { Insulin resistance: HOMA-IR } \\
\text { Insulin secretion: fasting insulin level, } \\
\text { fasting C-peptide, HOMA-B }\end{array}$ \\
\hline
\end{tabular}

Table 1. This table highlights that the published GWAS of response to antidiabetic agents used considerably smaller sample sizes, and therefore established less variants, than those GWAS of other diabetes mellitus-related traits. Given that more longitudinal data in large samples are increasingly available through large bioresources such as the UkBiobank, pharmacogenomic studies are expected to provide more insights into the genetic basis of various drug response phenotypes. 
Table 2. Replicated pharmacogenetic findings of antidiabetic agents

\begin{tabular}{|c|c|c|}
\hline Agent & Treatment Efficacy & ADRs \\
\hline Metformin & HbA1c reduction: $A T M^{39,62}$ & $\begin{array}{l}\text { Gastrointestinal side effects: } \\
\text { OCT1 }{ }^{69,73} \\
\text { Lactic Acidosis: (NA) }\end{array}$ \\
\hline Sulfonylureas & $\begin{array}{l}\text { HbA1c reduction: TCF7L2 } 2^{52-54} \\
\text { KCNJ11 }{ }^{55-57} \text { and CYP2C } 9^{49,51} \\
\text { Maintenance dose: CYP2C950 }\end{array}$ & Hypoglycaemia: CYP2C965 \\
\hline $\begin{array}{l}\text { Thiazolidinedione } \\
\text { (TZDs) }\end{array}$ & $\begin{array}{l}\text { HbA1c/Glucose reduction: } \\
P P A R G^{58-60}\end{array}$ & $\begin{array}{l}\text { troglitazone-induced } \\
\text { hepatotoxicity: GSTT1 } 168 \text { and } \\
\text { CYP2C } 19^{71}\end{array}$ \\
\hline $\begin{array}{l}\text { Gliptins } \\
\text { (DPP4i) }\end{array}$ & $\begin{array}{l}\text { HbA1c reduction (insulin } \\
\text { response): CTRB } 1 / 2^{61}\end{array}$ & $\begin{array}{l}\text { pancreatitis and liver } \\
\text { dysfunction: (NA) }\end{array}$ \\
\hline SGLT2i & NA & $\begin{array}{l}\text { genital and urinary tract } \\
\text { infections: (NA) }\end{array}$ \\
\hline
\end{tabular}

NA means no evidence has been published on the drug-outcome pair. 


\section{Author details}

Kaixin Zhou is a lecturer at the School of Medicine, University of Dundee, UK. Before this appointment, he was a Sir Henry Wellcome Fellow holding academic positions at the University of Dundee, University of Oxford, UK and University of Hong Kong, China. His current research interests include using the large-scale genomic data linked to electronic medical record, and pharmacoepidemiological approach to understand the biological mechanisms of drug action, drug-drug interaction, adverse drug reactions, and their relevance to stratified medicine.

Helle Krogh Pedersen is currently finishing her PhD studies at the department of Systems Biology, Technical University of Denmark, in Lyngby, Denmark. Her research focuses on multi-level systems biology approaches for integrating heterogeneous data sources in the context of diabetes mellitus.

Adem Y. Dawed received Master's Degree in Public health from Lund University, Sweden in 2014. He is currently a PhD student at the division of Cardiovascular and Diabetes Medicine, University of Dundee, UK. His research focuses on investigating clinical and genetic determinants of drug response in T2DM.

Ewan Pearson is a Professor in Diabetic Medicine and an Honorary Consultant in Diabetes and Endocrinology at the University of Dundee, UK,. He holds a new investigator award from the Wellcome Trust to investigate determinants of drug response in diabetes mellitus. His research interests include the phenotypic and genotypic determinants of drug response, the aetiology of young-onset diabetes mellitus and the mechanisms driving progression of this disease. 